\title{
A biodegradable thermo-responsive hybrid hydrogel: therapeutic applications in preventing the post-operative recurrence of breast cancer
}

\author{
Ying $\mathrm{Qu}^{1,3}$, Bing Yang $\mathrm{Chu}^{1,3}$, Jin Rong Peng ${ }^{1}$, Jin Feng Liao ${ }^{1}$, Ting Ting $\mathrm{Qi}^{1}$, Kun Shi ${ }^{1}$, Xiao Ning Zhang ${ }^{2}$, \\ Yu Quan $W^{1}{ }^{1}$ and Zhi Yong Qian ${ }^{1}$
}

Smart hydrogels that undergo structural changes in response to stimuli (for example, $\mathrm{pH}$, heat, light) have promising biomedical applications as delivery systems, especially for the locally controlled release of drugs. Early prevention of locoregional recurrence (LRR) is critical for patients who have undergone breast-conserving therapy. This work reports the preparation of a hybrid hydrogel system in which gold nanorods (GNRs) were doped into a thermally responsive hydrogel. A near-infrared (NIR) laser was used to trigger the release of loaded Doxorubicin (DOX) by utilizing the photothermal effect of GNRs to induce the contraction of the thermo-responsive hydrogels. In a 4T1 breast cancer model of the in vivo locoregional prevention of post-operative recurrence, we found that after NIR irradiation, DOX/GNR-embedded Methoxylpoly(ethylene glycol)-poly( $\varepsilon$-caprolactone)-acryloyl chloride (PECA)/glycidylmethacrylated chitooligosaccharide (COS-GMA)/N-isopropylacrylamide (NIPAm)/acrylamide (AAm) (PCNA) hydrogels (DOX-PCNA-GNR hydrogels) significantly reduced tumor recurrence to $16.7 \%$, compared with $50 \%$ for DOX-PCNAGNRs without NIR irradiation, $83.3 \%$ for PCNA-GNRs with NIR irradiation, 100\% for PCNA-GNRs without NIR irradiation, $83.3 \%$ for single systemic or local administration of Dox, $100 \%$ for intravenous DOX administration once or three times, and $100 \%$ for the blank control. This study demonstrates that these DOX-PCNA-GNR hybrid hydrogels with NIR-triggered thermo-responsive drug release exhibit great potential in preventing post-operation cancer relapse.

NPG Asia Materials (2015) 7, e207; doi:10.1038/am.2015.83; published online 14 August 2015

\section{INTRODUCTION}

Breast cancer is associated with a very high mortality ${ }^{1-3}$ and has profound implications for women's health. ${ }^{4,5}$ Various technologies have been proposed for breast cancer therapy, including surgical removal, site-specific radiotherapy or both. However, efficient inhibition of breast cancer relapse remains a clinical challenge, especially in patients who have undergone breast-conserving therapy. ${ }^{4,6}$ Post-operative recurrence is a fatal problem in breast cancer therapy. ${ }^{7}$

Locoregional recurrence (LRR) is the first site of tumor recurrence. This includes the soft tissue of the ipsilateral conserved breast or the chest wall, mastectomy scar or skin, lesions in the ipsilateral axillary lymph nodes, the extra-nodal soft tissue of the ipsilateral axilla or the ipsilateral internal mammary. ${ }^{8,9}$ Primary tumors and adjacent areas of the chest wall are at particularly high risk. Moreover, the early LRR of breast cancer after mastectomy can be easily predicted. Efficient inhibition of the early stage of recurrence can prevent distant tumor metastasis, which can in turn influence patient mortality. ${ }^{10,11}$ The localized characteristic of LRR makes local therapy suitable for the prevention of early recurrence. Adjuvant traditional chemotherapy, endocrine therapies and radiotherapy are the main methods used to reduce the risk of relapse in breast cancer patients. ${ }^{12-16}$ However, alternatives with lower toxicities and side effects remain urgently needed.

Recently, hydrogels have become popular drug-delivery vehicles. Hydrogels are among the best candidates for locally controlled drug release because of their low toxic and side effects. Hydrogels can undergo intelligent three-dimensional structural changes in response to environmental stimuli such as temperature, $\mathrm{pH}$, light and electrical fields. ${ }^{16-19}$ Hydrogels exhibit great potential for the local delivery of drugs, including small molecules and therapeutic proteins. ${ }^{20,21}$

Near-infrared (NIR) light is a relatively safe external stimulus that is able to penetrate several centimeters of human soft tissue with minimal damage. NIR light has been used as a stimulus for some nanosystems for the photothermal therapy (PTT) of cancer. PTT is a less invasive therapeutic approach than radiotherapy and chemotherapy, and it is an efficient approach to cancer therapy because tumor cells are highly sensitive to elevated temperatures.22-24 The potential of NIR-triggered PTT has spurred the development of NIR-responsive systems, including dyes and nanostructures. The first

\footnotetext{
${ }^{1}$ State Key Laboratory of Biotherapy and Cancer Center, West China Hospital, Sichuan University, and Collaborative Innovation Center for Biotherapy, Sichuan, China and ${ }^{2}$ School of Medicine, Tsinghua University, Beijing, China

${ }^{3}$ These authors contributed equally to this work.

Correspondence: Professor ZY Qian, State Key Laboratory of Biotherapy, Sichuan University, No. 17, Block 3, Southern Renmin Road, Chengdu 610041, China.

E-mail: anderson-qian@163.com

Received 9 April 2015; revised 24 May 2015; accepted 25 May 2015
} 
reported nanostructure used in NIR-induced PTT of cancer was silica-gold nanoshells. ${ }^{25}$ Based on the excellent inhibition of tumor growth by PTT, a variety of NIR-responsive nanostructures have been developed, such as gold nanorods (GNRs), gold nanocages, carbon nanotubes, graphene and Cu-based nanosystems. ${ }^{26-28}$ In addition to their PTT properties, NIR-responsive nanostructures can be used as

Table 1 Hydrogel samples prepared in this work

\begin{tabular}{lccc}
\hline Sample & PECA:COS-GMA:NIPAm:AAm (wt) & NIPAm (wt\%) & RI (\%) \\
\hline S-1 & $60: 12: 20: 8$ & 20 & 245.9 \\
S-2 & $45: 9: 40: 6$ & 40 & 409.5 \\
S-3 & $30: 6: 60: 4$ & 60 & 861.9 \\
\hline
\end{tabular}

Abbreviations: AAm, acrylamide; COS-GMA, glycidylmethacrylated chitooligosaccharide NIPAm, $N$-isopropylacrylamide; PECA, methoxylpoly(ethylene glycol)-poly(e-caprolactone)-acryloy chloride; Rl, response index. switches for controlled drug release. Surface coating with a thermally responsive polymer brush can result in NIR-controlled drug release from gold nanocages. ${ }^{29}$ Previous results have demonstrated that NIRresponsive nanostructures are promising heat generators for controlled drug release. Among the various NIR-responsive structures, GNRs are photothermal therapeutic agents that can inhibit cancer growth, and they have several distinct advantages, including small size, simple synthesis and tunable resonance from the red to the NIR, which has resulted in a great deal of research attention. ${ }^{30,31}$ Therefore, GNRs are ideal vehicles for heat generation. ${ }^{31-33}$

Furthermore, drug release from some intelligent hydrogels can be accelerated through hydrogel shrinkage induced by an external stimulus. This work combines PTT and chemotherapy to inhibit breast cancer relapse. The chemotherapeutic agent is released faster upon shrinkage of a thermally sensitive hydrogel due to the photothermal effect of GNRs under NIR irradiation.
COS-GMA

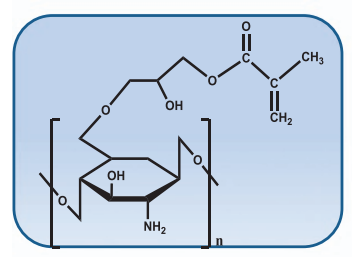

PECA

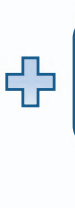<smiles>C=CC(=O)OCCCCCC(=O)OCCOC</smiles>

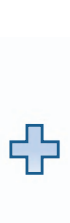
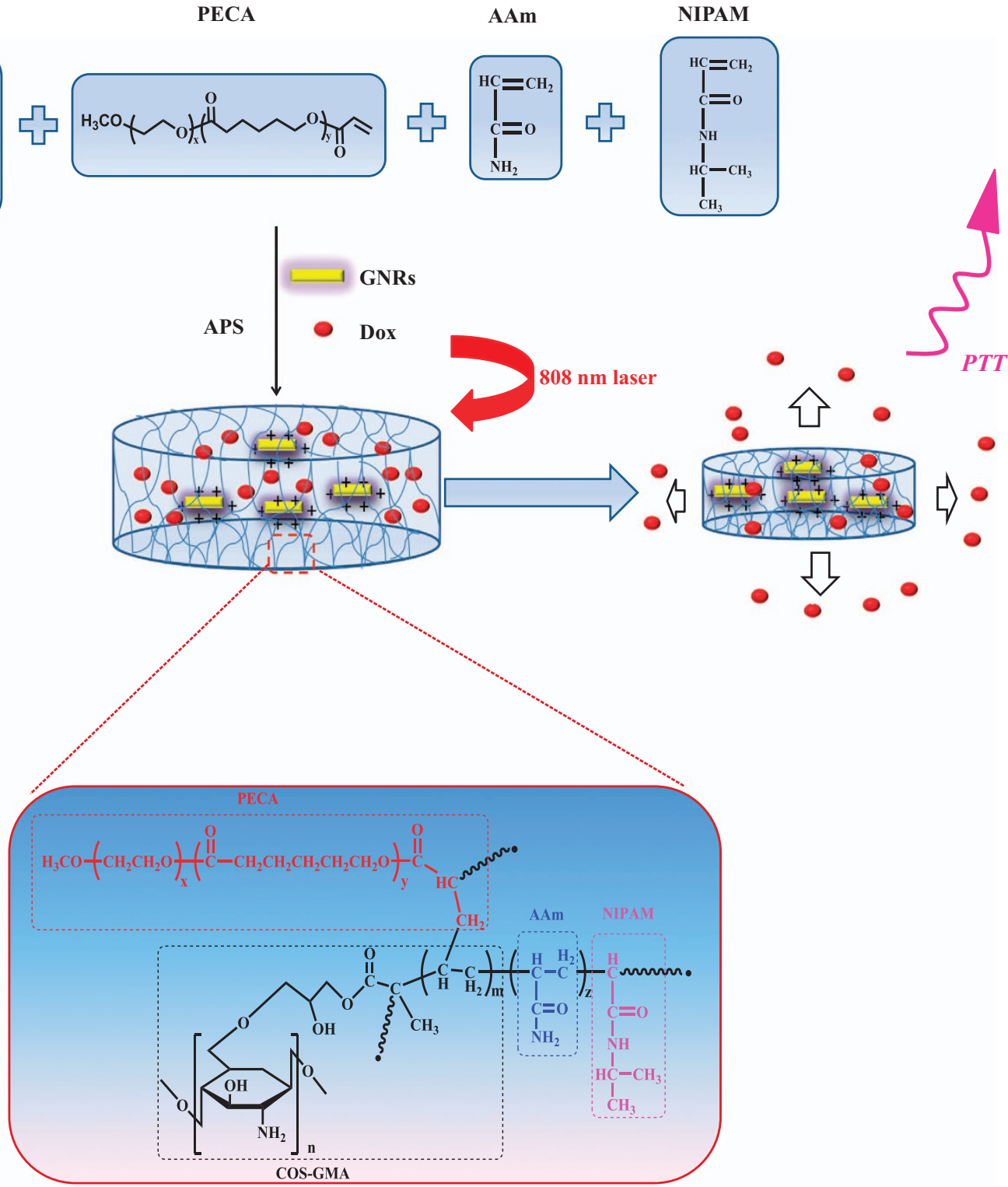

Scheme 1 Schematic illustrating that the reticulate cubic structure was more dense with smaller pore sizes and increased DOX release after $808 \mathrm{~nm}$ laser excitation. 
$\mathrm{N}$-isopropylacrylamide (NIPAm) is a monomer that can be used to synthesize thermally sensitive polymers. ${ }^{34}$ These polymers have been used widely to construct many thermo-sensitive systems, including hydrogels. Therefore, we integrated NIPAm with GNRs to develop a thermo-responsive hydrogel as the carrier for the chemophotothermal co-therapy of local breast cancer recurrence. Glycidylmethacrylated chitooligosaccharide (COS-GMA) and acrylamide (AAm) were chosen as co-monomers for GNR attachment. Methoxylpoly(ethylene glycol)-poly( $\varepsilon$-caprolactone)-acryloyl chloride (PECA) was used as a network spacer. The structural characterization, thermal responsiveness, in vitro cytotoxicity and in vivo inhibition of local breast cancer recurrence were studied in detail.

\section{EXPERIMENTAL PROCEDURES}

\section{Materials}

We purchased poly(ethylene glycol) methyl ether (MPEG, Mw $=2000$ ), $\varepsilon$-caprolactone $(\varepsilon-\mathrm{CL}), \mathrm{NIPAm}$, ammonium persulfate, Tin(II) 2-etheylhexanoate $\left(\mathrm{Sn}(\mathrm{Oct})_{2}\right)$, glycidyl methacrylate (GMA), sodium borohydride $\left(\mathrm{NaBH}_{4}\right)$, $\mathrm{L}(+)$-ascorbic acid $(\mathrm{Vc})$, and AAm from Sigma-Aldrich (St Louis, MO, USA). Cetyltrimethylammonium bromide and acid tetrachloroaurate(III) trihydrate $\left(\mathrm{HAuCl}_{4} \cdot 3 \mathrm{H}_{2} \mathrm{O}\right)$ were purchased from Sinopharm Co. Ltd (Beijing, China). Doxorubicin chloride (Doxorubicin, DOX) was supplied by Zhejiang Hisun Pharmaceutical Company, Zhejiang, China.

\section{Synthesis and purification of PCNA-GNR hydrogels}

The GNRs were synthesized according to the conventional procedure with some modifications. ${ }^{35}$ Briefly, Au seeds were first synthesized. An aqueous $0.01 \mathrm{M}$ solution of $\mathrm{HAuCl}_{4} \cdot 3 \mathrm{H}_{2} \mathrm{O}$ and $7.5 \mathrm{ml}$ of $0.1 \mathrm{M}$ cetyltrimethylammonium bromide was gently mixed. Then, $600 \mu \mathrm{l}$ of $0.1 \mathrm{M}$ ice-cold $\mathrm{NaBH}_{4}$ solution was added with stirring for $2-3 \mathrm{~min}$. A brown-yellow seed solution was obtained and kept at room temperature for $2-5 \mathrm{~h}$ before use.

The GNRs were prepared by seed-mediated growth. First, $100 \mathrm{ml}$ of $0.1 \mathrm{M}$ cetyltrimethylammonium bromide, $2.04 \mathrm{ml}$ of $24 \mathrm{mM} \mathrm{HAuCl}_{4}, 2 \mathrm{ml}$ of $0.5 \mathrm{M}$ $\mathrm{H}_{2} \mathrm{SO}_{4}, 0.9 \mathrm{ml}$ of $0.01 \mathrm{M} \mathrm{AgNO}, 0.8 \mathrm{ml}$ of $0.1 \mathrm{M} \mathrm{L}$-ascorbic acid and $240 \mu \mathrm{l}$ of Au seed solution were mixed under gentle stirring. The solution was kept at room temperature overnight without stirring. Then, GNRs were purified by centrifugation

(12 000 r.p.m., $10 \mathrm{~min}$ ) and washed twice with distilled water.

The MPEG-PCL copolymer was synthesized by ring-opening copolymerization based on our previous report. ${ }^{36}$ After polymerization, the MPEG-PCL was further reacted with acryloyl chloride at $40^{\circ} \mathrm{C}$ to obtain the macromonomer MPEG-PCL-AC (PECA). A COS-GMA was synthesized by conjugating glycidylmethacrylate to the hydroxyl groups of $\operatorname{COS}^{37}$ The ${ }^{1} \mathrm{H}$-Nuclear
Magnetic Resonance Spectroscopy spectrum of the copolymer is presented in Supplementary Figure S1 and S2 in the Supplementary Information.

The PECA/COS-GMA/NIPAm/AAm (PCNA) hydrogel was synthesized by heat-initiated free radical polymerization using ammonium persulfate as a heat initiator. The mixture of PECA, COS-GMA, NIPAm, AAm and ammonium persulfate was dissolved in water (Table 1). The solution was then heated at $50^{\circ}$ $\mathrm{C}$ for $1 \mathrm{~h}$. The hydrogel was dialyzed for 3 days, and the water was refreshed every day. The purified hydrogels were freeze-dried and kept in airtight bags before use. The DOX/GNR-embedded PCNA hydrogel (DOX-PCNA-GNRs) was synthesized by the same method, but the monomers were dissolved in the GNR solutions and/or the GNR-DOX mixture.

\section{PCNA hydrogel characterization}

Fourier Transform Infrared Spectrometer spectra $(\mathrm{KBr})$ of the hydrogel sample and the macromonomers were recorded on a Nicolet 200SXV spectrophotometer (Nicolet, Thermo Scientific, Waltham, MA, USA).

Scanning electron microscopy (SEM) was used to investigate the morphology of the PCNA hydrogel. The hydrogels were immersed at 25 or $40^{\circ} \mathrm{C}$, frozen in liquid nitrogen and then lyophilized. The hydrogels were then sputtered with gold before observation with a JEOL SEM (JSM-5900LV, JEOL, Tokyo, Japan).

The hydrogel samples were immersed in aqueous media at 25 or $45^{\circ} \mathrm{C}$. At predetermined time points, the samples were removed and the surplus surface water was removed by filter paper. The swelling ratio can be determined by ${ }^{36}$

Swelling ratio $(\mathrm{SR})=\mathrm{W}_{\mathrm{t}} / \mathrm{W}_{0 *} 100 \%$,

where $W_{\mathrm{t}}$ is the wet weight of the hydrogels and $W_{0}$ is the initial dry weight at predetermined times. The swelling ratio after immersion in aqueous solutions for $30 \mathrm{~h}$ is defined as the equilibrium swelling ratio.

The dynamic swelling/deswelling behavior of the hydrogels was studied by immersing defined amounts of hydrogels in aqueous media at 25 and $45^{\circ} \mathrm{C}$, alternating between these temperatures.

The dried hydrogels were immersed in an aqueous medium at $25^{\circ} \mathrm{C}$, and their wet weights were recorded at $0.5,1,2,4,8,10$ and $24 \mathrm{~h}$. The medium was then replaced by another batch of fresh medium at $45^{\circ} \mathrm{C}$, and this process was repeated. The experiment was repeated for three cycles. A response index (RI) was defined to characterize the thermosensitivity of the hydrogels. ${ }^{38}$

$$
\mathrm{RI}(\%)=\mathrm{SR}_{\left(25^{\circ} \mathrm{C}\right), \mathrm{t}=24 \mathrm{~h}}-\mathrm{SR}_{\left(45^{\circ} \mathrm{C}\right), \mathrm{t}=48 \mathrm{~h}}
$$

\section{Photothermal effects of PCNA-GNR hydrogels}

The hydrogel was irradiated from the right side with an 808-nm laser at a power density of $2.5 \mathrm{~W} \mathrm{~cm}^{-2}$ for $5 \mathrm{~min}$, and the temperature was recorded by a Fluke TI32 Infrared (IR) thermal camera (Infrared Cameras, Fluke, Avery, WA, USA) until the sample reached room temperature.

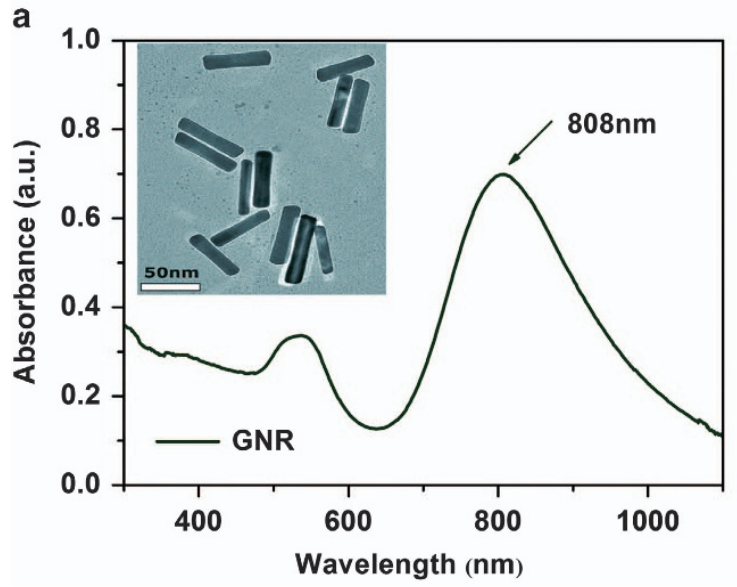

b

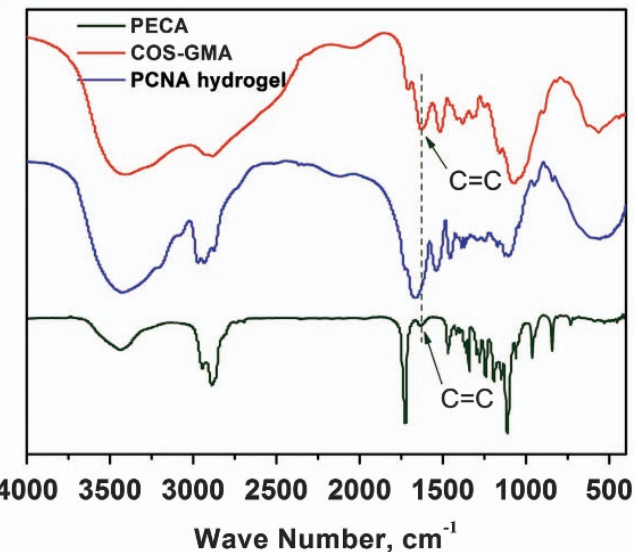

Figure 1 (a) UV-vis absorption spectra of GNRs. Inset: TEM; (b) FTIR spectra of PECA, COS-GMA macromonomer and PCNA hydrogel. 
Cytotoxicity assay of PCNA-GNR hydrogels

A PCNA-GNRs hydrogel was extracted using DMEM for $24 \mathrm{~h}$. Sequential dilutions of the stock solution were prepared to vary the concentrations of the leachates. 3T3 cells were seeded in 96-well plates after growing for $24 \mathrm{~h}$ in $100 \mu$ leachates at different concentrations $(6.25,12.5,25,50$ and $100 \% ; n=3)$. At predetermined times, $20 \mu \mathrm{l}$ of MTT was added to the wells, and the cells were further incubated for another $4 \mathrm{~h}$. The formazan was dissolved in $150 \mu \mathrm{l}$ DMSO, and the absorbance was measured at $570 \mathrm{~nm}$. These experiments were repeated three times.

In vivo degradation study of the hydrogel

The in vivo biocompatibility of the hydrogel with GNRs was investigated by implanting the materials into the subcutaneous tissues of mice. Then, the mice were killed at predetermined intervals. The materials were implanted, and the surrounding tissues were removed and analyzed by histology. The biocompatibility was studied by hematoxylin and eosin staining.

\section{IR thermal imaging}

To facilitate observation, we implanted the PCNA-GNR hydrogels subcutaneously into the backs of anesthetized mice. At $24 \mathrm{~h}$ after implantation, the hydrogel area was irradiated with an $808-\mathrm{nm}$ laser at a power density of 2.5 $\mathrm{W} \mathrm{cm}{ }^{-2}$ for $5 \mathrm{~min}$. The temperatures of the back tissues were recorded during NIR irradiation. At $48 \mathrm{~h}$ after implantation, the same hydrogel area was exposed again using the same NIR irradiation procedure. The temperature distribution of the mice was obtained with a Fluke TI32 IR thermal camera (Infrared Cameras).

\section{In vitro drug release behavior}

To measure the effects of these temperature changes on DOX release, the DOXloaded hydrogel with GNRs was immersed in $37^{\circ} \mathrm{C}, \mathrm{pH}=5.0$ and 7.4 phosphate-buffered saline with stirring at 100 r.p.m. At predetermined intervals, the release media were removed and replaced with fresh buffer. Some samples were irradiated with an $808-\mathrm{nm}$ NIR laser at $2.5 \mathrm{~W} \mathrm{~cm}^{-2}$ for $5 \mathrm{~min}$. The concentration of DOX was determined by absorbance spectroscopy.

\section{In vivo inhibition of locoregional breast cancer recurrence}

All animal procedures were carried out according to the standard procedures of the State Key Laboratory of Biotherapy at Sichuan University, China. Balb/C mice were supplied by Beijing HFK Bioscience Co. Ltd, Beijing, China (female, 4-6 weeks old). Then, $10^{6} 4 \mathrm{~T} 1$ cells per mouse were injected into the right thoracic mammary fat pad to produce orthotopic primary tumors. Generally, a small incision was made after the mice were anesthetized. The tumor was completely removed when it reached $200 \mathrm{~mm}^{3}$. The covering and surrounding skin of the tumors was preserved to ensure a high rate of recurrence in untreated mice. Four groups of mice were implanted with DOX-PCNA-GNR hydrogels (Group 1 and Group 2) and PCNA-GNRs without DOX (Group 3 and Group 4), respectively. The other four groups were injected with free DOX $(250 \mu \mathrm{g})$ once or thrice intravenously or once subcutaneously. Normal saline

a

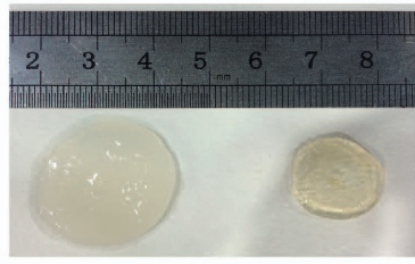

b

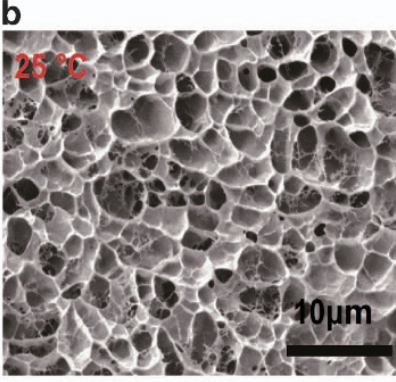

d

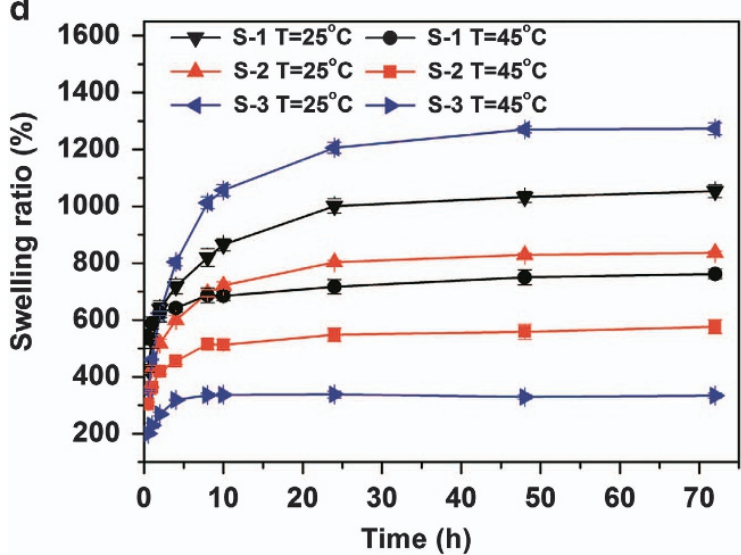

c
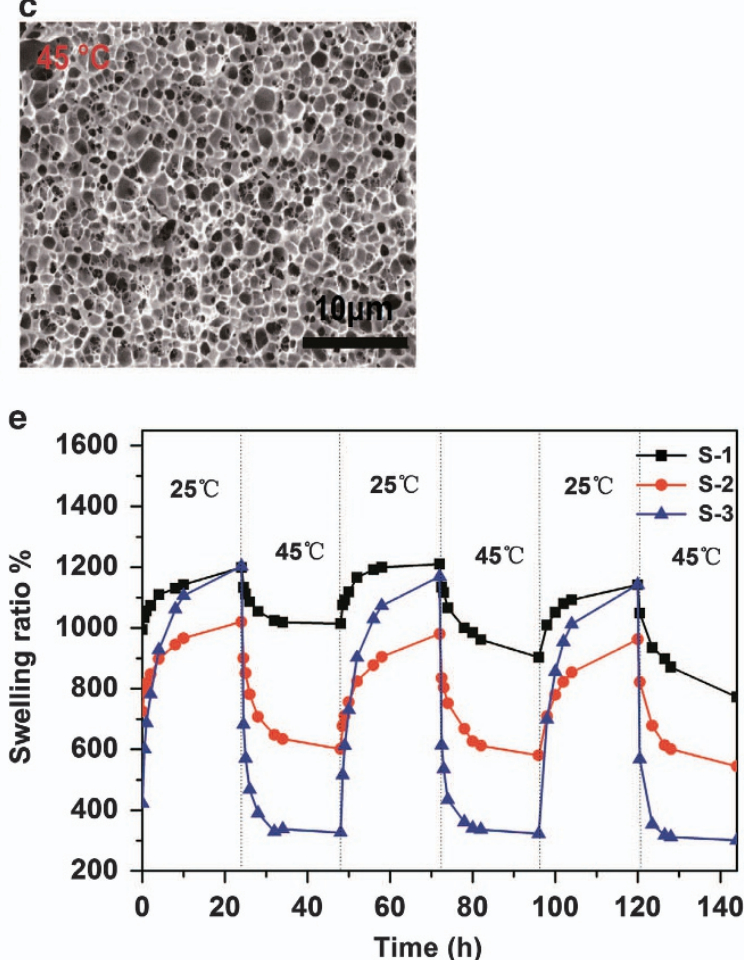

Figure 2 (a) A photo of hydrogel swelling without GNRs at $25^{\circ} \mathrm{C}$ (left) and $45^{\circ} \mathrm{C}$ (right); SEM images of hydrogels at $25^{\circ} \mathrm{C}$ (b) and $45^{\circ} \mathrm{C}$ (c); (d) Swelling behavior of hydrogels in aqueous medium at 25 and $45^{\circ} \mathrm{C}$; (e) The dynamic swelling/deswelling behavior of hydrogels in aqueous medium at 25 and $45^{\circ} \mathrm{C}$ at 24-h intervals. 
was injected subcutaneously as a control. All mice were monitored every other day for body weight and tumor recurrence. Mice were killed when the tumors reached $14-16 \mathrm{~mm}$ or when the mice became moribund.

\section{Histopathological study}

After treatment, the heart, liver, spleen, lung and kidney of all groups were subjected to histopathological analysis, including hematoxylin and eosin staining.

\section{Statistical analysis}

Mean values and standard deviations were calculated for the amount of drug released from PCNA hydrogels with GNRs and for the swelling, IC50, tumor weight and body weight. Differences were considered as significant at $P$-values $<0.05$. Statistical analysis was performed using SPSS 11.0 software (SPSS Inc., Chicago, IL, USA).

\section{RESULTS AND DISCUSSION}

Synthesis and characterization of GNR-loaded hydrogels The GNR-loaded hydrogel (Scheme 1) was composed of GNRs and PCNA hydrogel copolymerized with PECA (Supplementary Figure S1 in the Supplementary Information), COS-GMA (Supplementary Figure S2 in the Supplementary Information), NIPAm and AAm. Figure 1a demonstrates that the synthesized GNRs have a uniform morphology. The GNRs exhibited strong absorbance in the NIR region with a peak at $808 \mathrm{~nm}$, which suggests that the GNRs were successfully prepared.

To characterize the PCNA hydrogel, we first prepared empty PCNA hydrogels. The FTIR spectra of PECA, COS-GMA and PCNA hydrogels are presented in Figure 1b. The absorption band at $\sim 1620 \mathrm{~cm}^{-1}$ is attributed to the $\mathrm{C}=\mathrm{C}$ stretching vibration and can be clearly observed in the PECA and COS-GMA FTIR spectra. This band is absent in the PCNA hydrogel spectrum, which suggests that PECA and COS-GMA were copolymerized into the structure of the PCNA hydrogel.

The monomers were dissolved in the GNR aqueous solutions so that the GNRs could be well distributed within the hydrogels. The amount of GNRs loaded in the PCNA freeze-dried hydrogel was determined to be $0.5 \%$ by ICP-AES. The porous structure of the hydrogel was clearly observed by SEM (Figures $2 \mathrm{~b}$ and c).

Water adsorption is a key property of hydrogels. We measured the water adsorption of the GNR-loaded PCNA hydrogel at 25 and $45^{\circ} \mathrm{C}$ (Figure 2d). As shown in Figure 2d, the equilibrium swelling ratios of the hydrogels at $25^{\circ} \mathrm{C}$ are much higher than those at $45^{\circ} \mathrm{C}$. The equilibrium swelling ratios at $25^{\circ} \mathrm{C}$ increased with increasing NIPAm contents in the S-1, S-2 and S-3 hydrogels. These ratios were relatively a

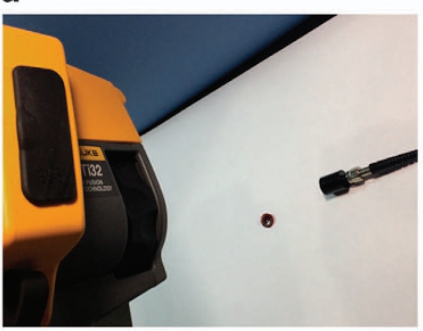

b

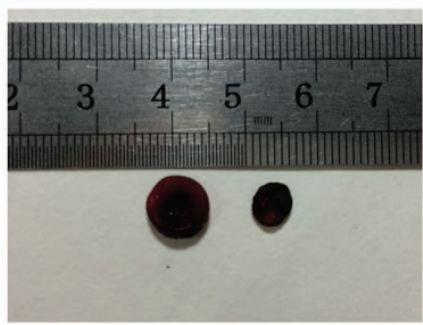

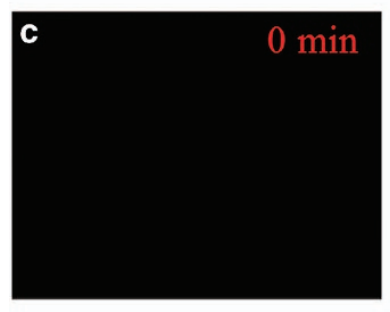
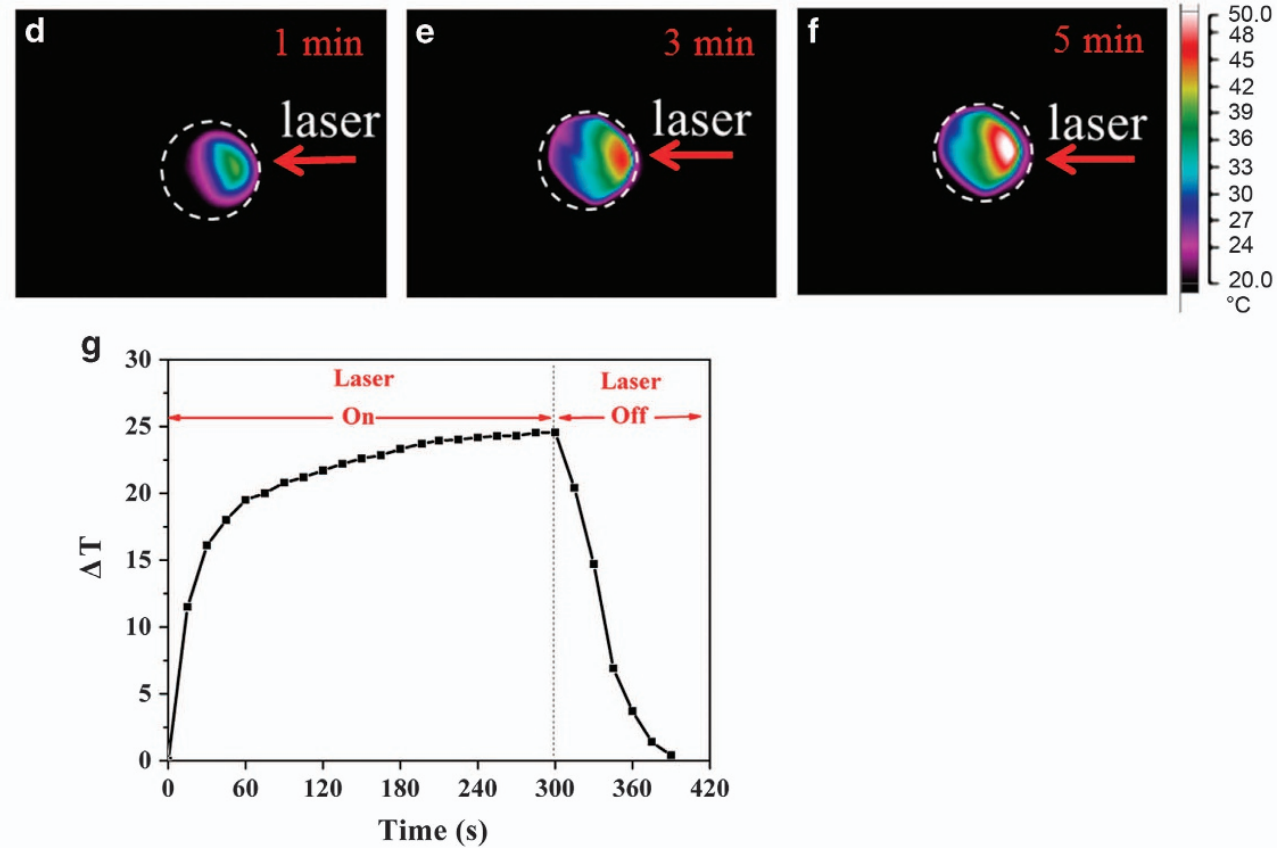

Figure 3 (a) The laser was irradiated from the right side of the hydrogel samples and the temperature was obtained vertically by a Fluke TI32 IR thermal camera; (b) DOX-PNCA-GNR hydrogels before (left) and after (right) $808 \mathrm{~nm}$ laser irradiation; near infrared thermal imaging of PCNA-GNR hydrogels under $2.5 \mathrm{~W} \mathrm{~cm}^{-2}$ irradiation at $808 \mathrm{~nm}$ for (c) $0 \mathrm{~min}$, (d) $1 \mathrm{~min}$, (e) $3 \mathrm{~min}$ and (f) $5 \mathrm{~min}$. NIR laser irradiation increased the temperature of the PCNA-GNRs hydrogel to $50^{\circ} \mathrm{C}$ in $5 \mathrm{~min}$; $(\mathrm{g})$ the change in temperature under $2.5 \mathrm{~W} \mathrm{~cm}^{-2}$ irradiation at $808 \mathrm{~nm}$. 
lower at $45^{\circ} \mathrm{C}$. These results indicate that the PCNA hydrogels exhibit significant water adsorption that can be tuned by adjusting the feed ratios of the monomers.

We then evaluated the dynamic swelling/deswelling behavior (temperature sensitivity) of PCNA hydrogels, including their equilibrium-reswelling behavior. The GNR-loaded PCNA hydrogels were alternately immersed at 25 and $45^{\circ} \mathrm{C}$. The swelling ratios of the hydrogels were recorded at predetermined time points. The results are presented in Figure 2e. Figure $2 \mathrm{e}$ shows that as the NIPAm dose increased, the hydrogel shrinkage at $45^{\circ} \mathrm{C}$ was enhanced. This result indicates that the thermally responsive property of the NIPAm hydrogel is content dependent. Furthermore, Figure 2e shows that the thermal response of the hydrogel remains constant, even after three cycles. This finding demonstrates that the hydrogel exhibits a sensitive thermal response and that the network structure is robust.

We further characterized the thermal response of the hydrogel using SEM. Figures $2 b$ and $c$ show SEM images of the hydrogel sections

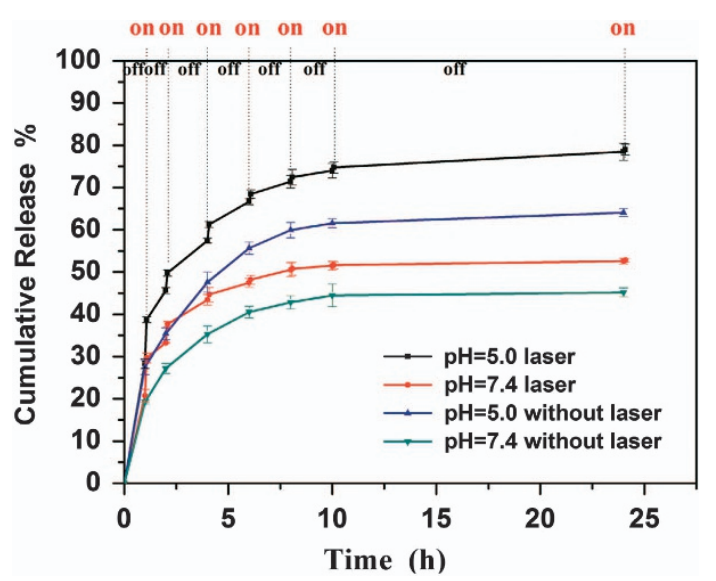

Figure 4 DOX-release profiles in the presence and absence of NIR laser at $\mathrm{pH} 5.0$ and 7.4 .
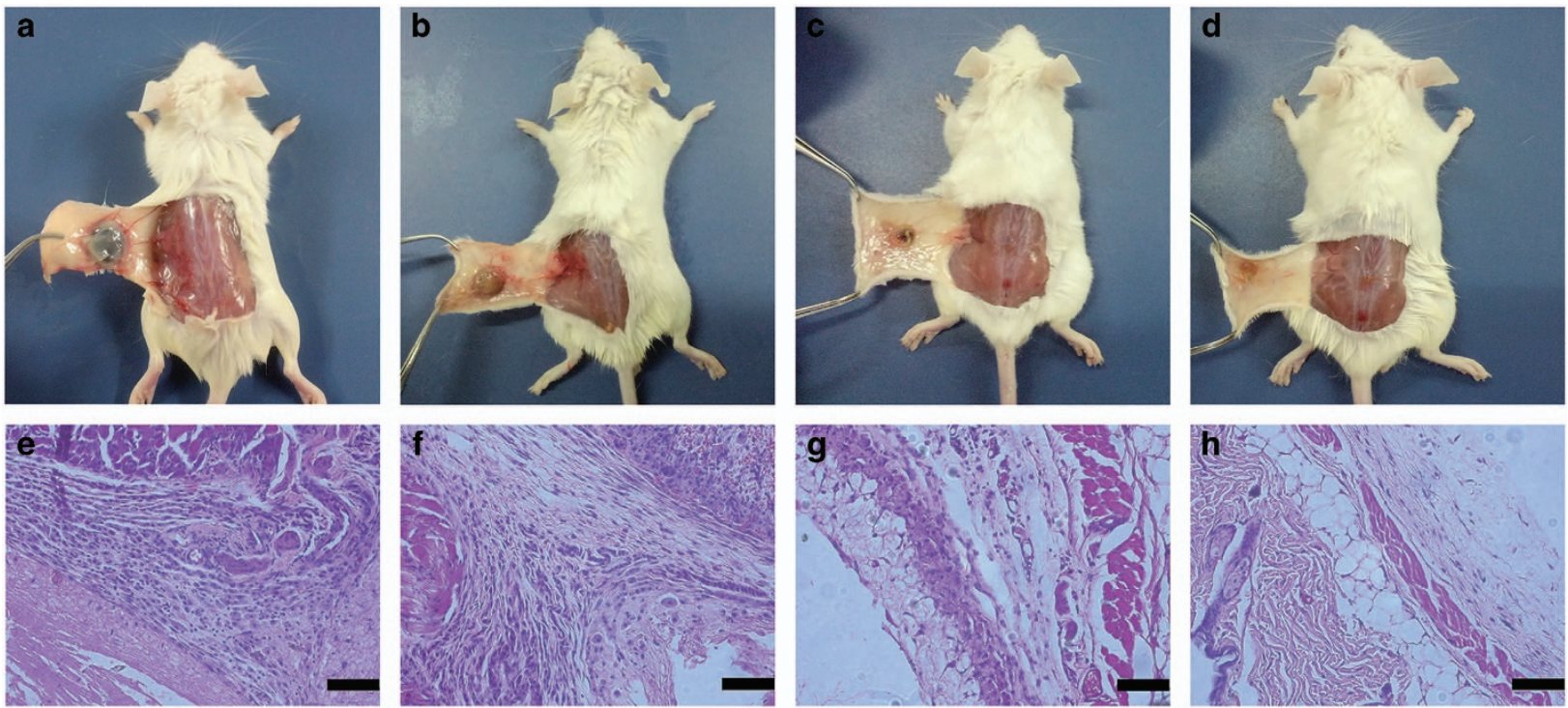

Figure 6 In vivo persistence and histology sections from subcutaneous rat tissue after implantation of PCNA-hydrogel composites for different periods. Images in panels a (e), b (f), c (g) and $\mathbf{d}$ (h) were captured at 7, 14, 28 and 35 days, respectively (scale bar: $200 \mu \mathrm{m}$ ). 
hydrogel, the GNRs were well distributed in the hydrogel. If the facula were larger than the hydrogel and the laser was irradiated vertically, then the temperature would be constant through the depth of the hydrogel.

After irradiation, the volume of the hydrogel was smaller than before irradiation (Figure 3b). The swelling/deswelling ratio of the DOX-PCNA hydrogel reached 550\%.

These results demonstrate that the PCNA-GNRs hydrogel is also a promising candidate for topical photothermal cancer therapy and that it may serve as a local drug delivery system capable of light-triggered controlled release.

\section{In vitro release behavior}

The release of DOX from the PCNA-GNR hydrogels can be readily controlled by NIR laser irradiation and $\mathrm{pH}$ manipulation. Within $5 \mathrm{~min}$ after the first NIR laser irradiation at $2.5 \mathrm{~W} \mathrm{~cm}^{-2}$ output power, the cumulative amount of DOX release increased from 28.3 to $38.7 \%$. This was much higher than that observed without irradiation at $\mathrm{pH}$ 5.0. The release was significantly reduced when the NIR laser was switched off over the next $1 \mathrm{~h}$. Similar results were observed when the laser treatment was repeated in other treatment cycles. The solubility of DOX increases as the $\mathrm{pH}$ value decreases, which occurs in the extracellular space within tumor tissues and in lysosomes, which have lower $\mathrm{pH}$ values than the cytosol. ${ }^{26}$ More DOX was released from the hydrogels with GNRs at $\mathrm{pH} 5.0$ than at $\mathrm{pH}$ 7.4. A cumulative release of $48 \%$ DOX was achieved in phosphate-buffered saline at $\mathrm{pH} 7.4$, but $77 \%$ was released at pH 5.0 in $24 \mathrm{~h}$ (Figure 4). Thus, these smart hydrogels demonstrate a synergistic effect, with the release of anticancer drugs controlled by the dual stimuli of NIR irradiation and $\mathrm{pH}$.

\section{Cytotoxicity}

The cytotoxicity of PCNA-GNR hydrogels was studied by the MTT assay. As the results in Figure 5 indicate, all of the leachates from the PCNA-GNR hydrogels had low toxicity over the concentration range tested here. The cells are able to proliferate well in the presence of hydrogel leachate, with proliferation similar to the negative control ( $0 \%$ leachate). These results demonstrate that the PCNA-GNRs are relatively safe to healthy cells.

Degradation study of the hydrogel in vivo

Due to the inclusion of COS, we expected the PCNA hydrogel to be biodegradable in vivo. The in vivo biocompatibility of hydrogels with

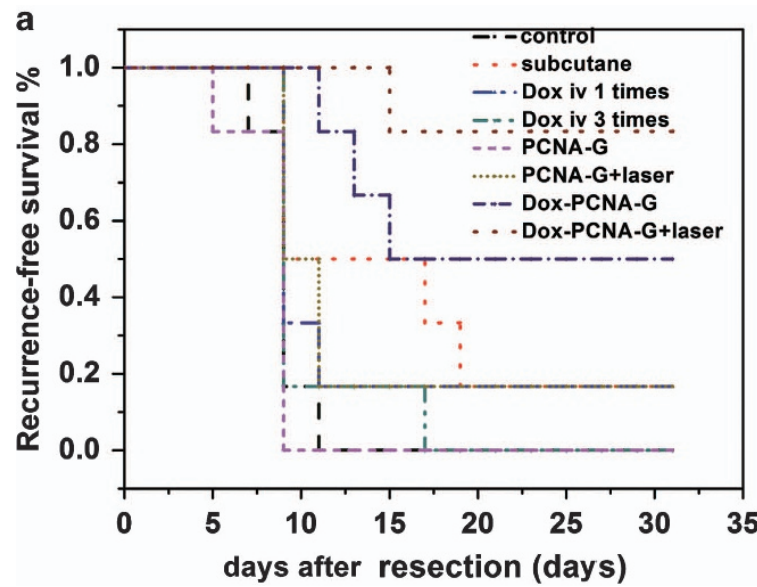

GNRs was investigated by subcutaneously implanting the composite into the mice, and then killing the mice at 7, 14, 28 and 35 days after implantation. By visual observation, we found that clear degradation occurred in 14 days and that the hydrogel completely disappeared in 35 days. This result indicates that the PCNA hydrogel can be biologically metabolized. We further investigated the induction of inflammation by the hydrogel by histological observation. As shown in Figure 6, for the first 7 days, the implants remained relatively intact, and a thin, translucent tissue capsule was observed. Some inflammatory cells were also found, which implies that the
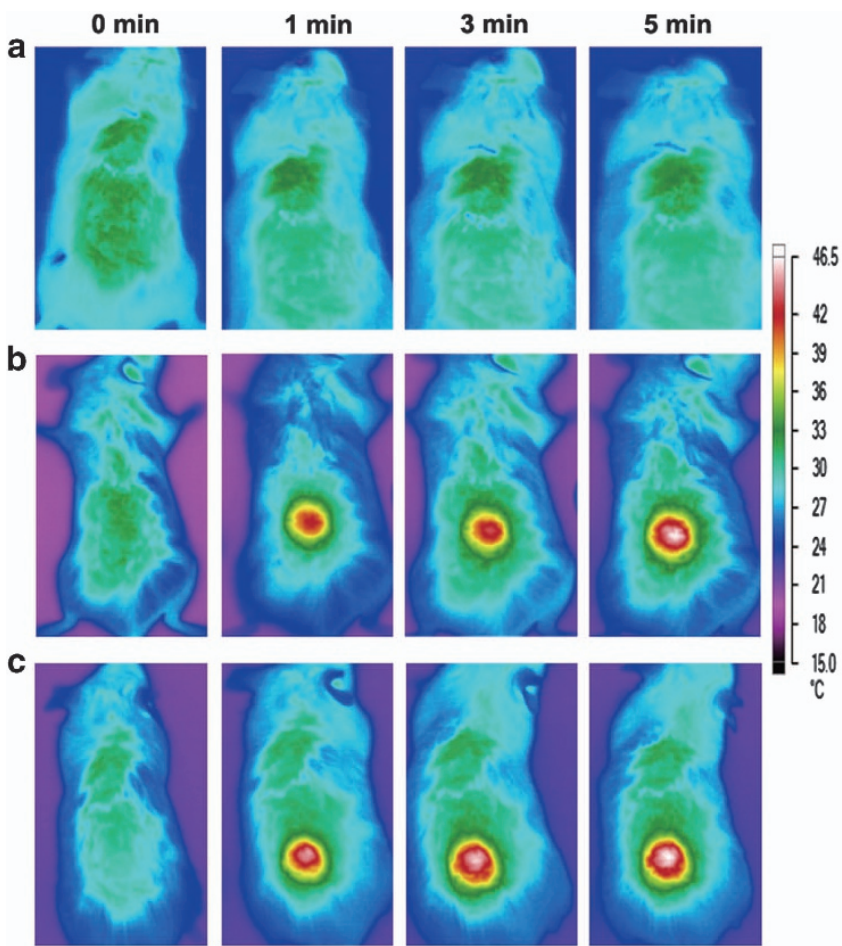

Figure 7 (a) Infrared thermal images of the control, with no change in temperature observed; (b) $24 \mathrm{~h}$ after implantation, $5 \mathrm{~min}$ of irradiation increased the temperature to $46^{\circ} \mathrm{C}$; (c) $48 \mathrm{~h}$ after implantation, $1 \mathrm{~min}$ of irradiation increased the site temperature to $46^{\circ} \mathrm{C}$; when the irradiation time was increased, the area stayed at $46^{\circ} \mathrm{C}$, but the heat spread across a larger area. Irradiation times are $0,1,3$ and $5 \mathrm{~min}$.

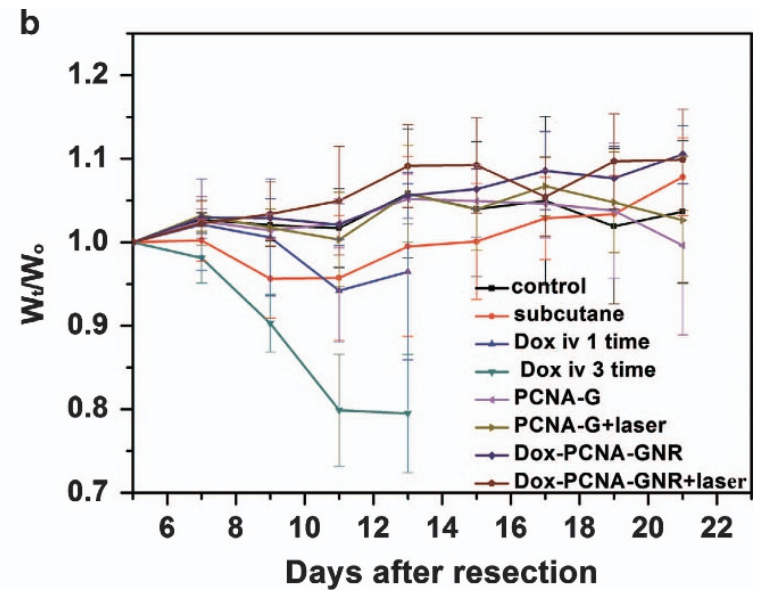

Figure 8 (a) In vivo breast cancer locoregional recurrence; (b) body weight variation after treatment. $P<0.05$. 
PCNA hydrogel induced a slight inflammatory reaction. However, no inflammation was observed on the 35th day. These results demonstrate that the GNR-loaded PCNA hydrogel is safe, biodegradable and biocompatible.

Because implanted materials should have excellent biocompatibility and suitable biodegradability, the hydrogel in this study was primarily composed of MEG-PCL, COS-GMA, NIPAm and N,N'-methylenebisacrylamide (BIS). Previous reports have demonstrated that MPEG-PCL and COS-GMA are biodegradable and biocompatible both in vitro and in vivo. ${ }^{39,40}$ The degradation of PCNA-GNR hydrogels may occur at its PCL block through the cleavage of its ester bonds. This may lead to a decrease in molecular weight and changes in chemical composition. We expect that the GNRs can be cleared from the body by excretion. Throughout the experiments, there were no signs of hematoma or purulence, which suggests a lack of observable toxicity of the hydrogels to the surrounding tissues.

\section{In vivo photothermal performance}

Twenty-four hours after implantation, the subcutaneous GNRloaded PCNA hydrogels were exposed to an 808-nm laser beam at $2.5 \mathrm{~W} \mathrm{~cm}{ }^{-2}$ for $5 \mathrm{~min}$. The temperature increased to $46^{\circ} \mathrm{C}$, but not for blank PCNA hydrogels. This result demonstrates that the PCNA-GNRs are efficient agents for in vivo photothermal treatment. After another $24 \mathrm{~h}$, the subcutaneous hydrogels were exposed again to the same laser intensity and time. After $1 \mathrm{~min}$, the site temperature increased to $46^{\circ} \mathrm{C}$. As the NIR irradiation time was increased, the focus area stayed at $46^{\circ} \mathrm{C}$, but the heat spread across a larger area (Figure 7). The temperature of the mice without implanted
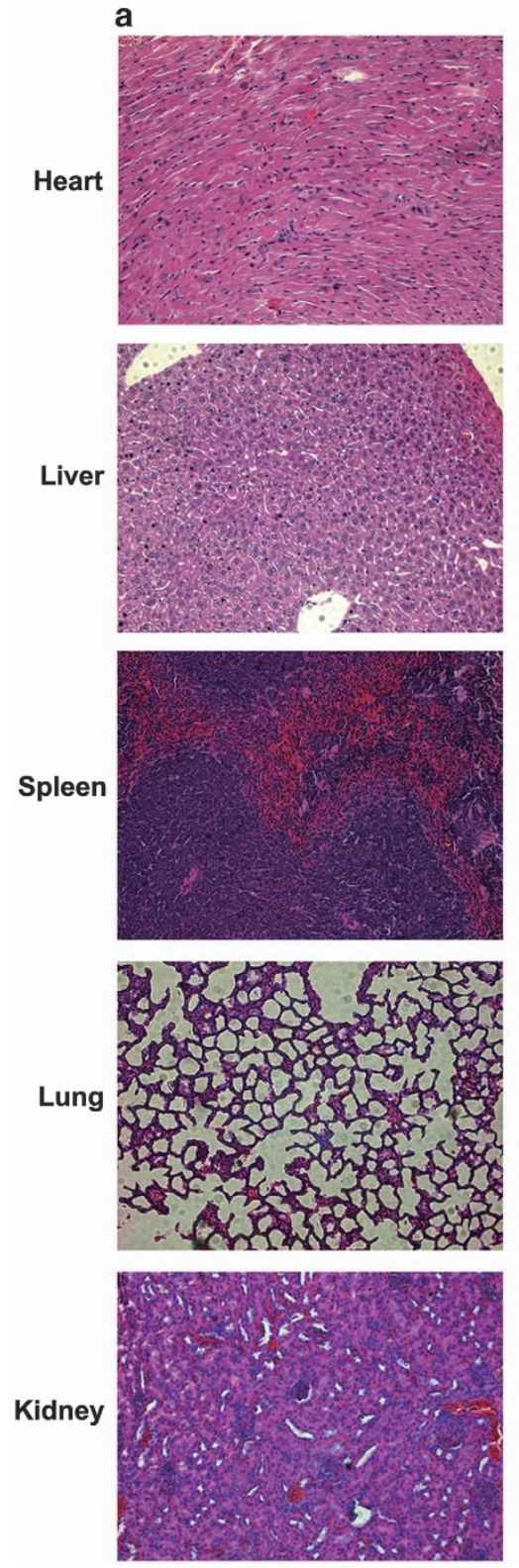

b
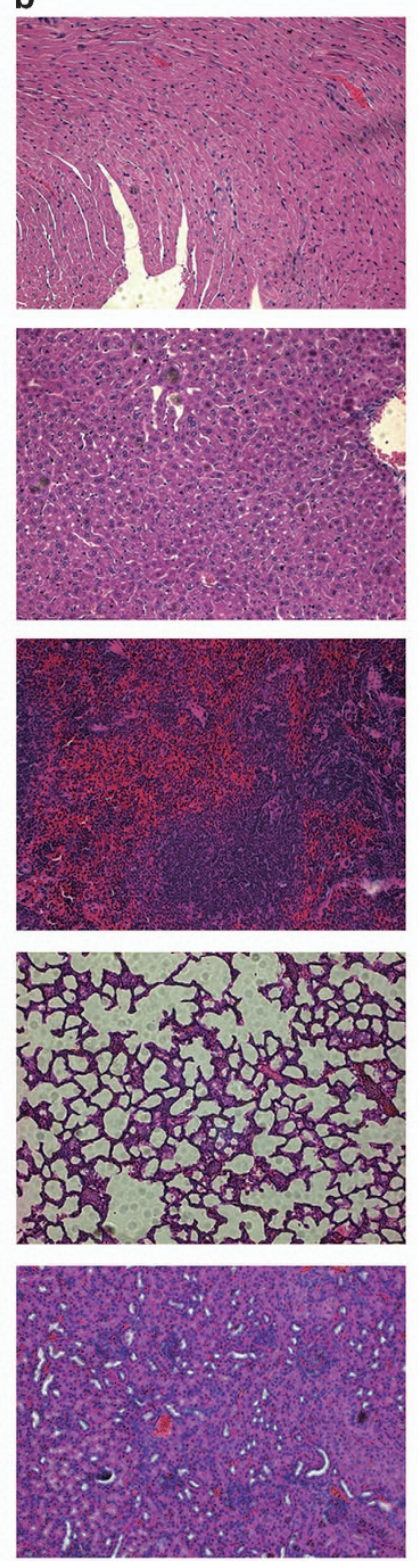

c
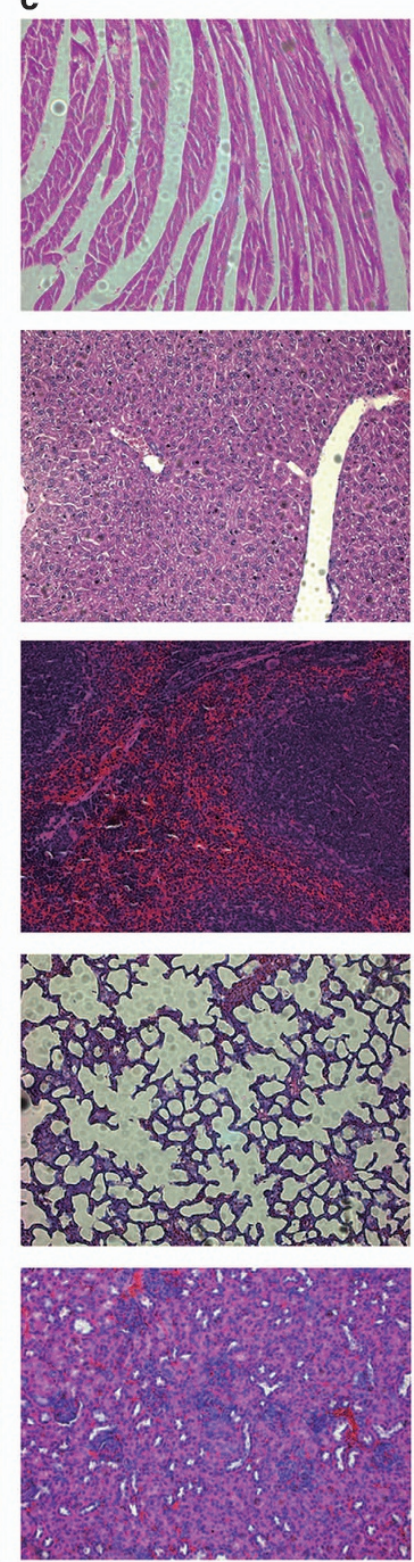

Figure 9 H\&E stained images of the heart, liver, spleen, lung and kidney. Representative H\&E stained images of NS (a), DOX-PCNA-GNRs+laser (b) and free DOX (intravenous injection) (c). No cardiotoxicity was observed in the DOX-PCNA-laser treated group, but obvious toxicity was observed in the free DOX treated group (intravenous injection). No lesions were observed in the liver, spleen, lung or kidney. 
PCNA-GNR hydrogels did not increase after NIR irradiation. This result indicates that the PCNA-GNRs system is stable in vivo and can be repeatedly irradiated.

\section{In vivo locoregional breast cancer recurrence}

Based on the in vitro drug release behavior, we expected that DOX-PCNA-GNR hydrogels could effectively prevent the local recurrence of breast cancer post resection. The in vivo results of locoregional tumor recurrence revealed that DOX-PCNA-GNR hydrogels achieved better inhibition of recurrence than did the other groups. The period of LRRs lasted for 32 days. During this time, only one of the six mice treated with DOX-PCNA-GNRs and laser irradiation developed locoregional tumor recurrence (16.7\%), three of six mice treated with DOX-PCNA-GNRs without laser irradiation (50\%) and five of six mice with DOX locally administered and PCNAGNRs without drugs with laser irradiation, and intravenously injected with DOX once with the dosage of $250 \mu \mathrm{g}$ did develop tumors $(83.3 \%)$. All six mice in the control groups (PCNA-GNRs with no laser, empty hydrogels with no drugs and those with intravenously injected DOX three times) steadily developed tumors (Figure 8a). The recurrence rate in vivo was significantly suppressed by the DOXPCNA-GNRs hydrogel.

DOX is an excellent anticancer drug, but has some serious side effects. As shown in Figure 8b, the systemic toxicity of free DOX given subcutaneously and injected one or three times resulted in the decrease in body weight. There was no difference in the body weight of mice between the DOX-PCNA-GNR treated group and the other groups including the control group and the PCNA-GNRs without the DOX group. By histopathology, no cardiotoxicity was found in the DOX-PCNA-laser treated group (Figure 9). However, the cardiotoxicity was obviously visible in the free DOX treated group (intravenous injection). After treated with the DOX-PCNA-GNRs, we did not find out lesions in the liver, spleen, lung and kidney. This indicated that the hydrogels reduced the toxicity of DOX, and the PCNA-hydrogels system has better performance than free DOX and has alleviated some side effects of DOX.

The efficient inhibition of locoregional tumor recurrence by the DOX-PCNA-GNRs hydrogel was due to the synergetic effects of PTT and chemotherapy. The combination of PTT and chemotherapy significantly inhibited tumor growth. ${ }^{26}$ The results indicated that the PCNA-GNR hydrogel is a suitable carrier for the inhibition of postoperation locoregional tumor recurrence via combination treatment with PTT and chemotherapy.

\section{CONCLUSION}

In summary, we have successfully synthesized a thermoresponsive (PCNA) hydrogel by heat-initiated free radical polymerization. The DOX-PCNA-GNRs hybrid hydrogel prepared here facilitates chemo-photothermal co-therapy and prevents local breast cancer recurrence. When the PCNA-GNRs were irradiated, the temperature of the system increased, causing a decrease in the mesh size of the hydrogel, thereby accelerating the release of loaded drug. The DOX-PCNA-GNRs hydrogel effectively prevented breast cancer recurrence after primary tumor resection in a mouse model. This local system for the controlled administration of combination chemo-photothermal therapy decreased non-selective systemic toxicity.

\section{CONFLICT OF INTEREST}

The authors declare no conflict of interest.

\section{ACKNOWLEDGEMENTS}

This work was financially supported by the National Natural Science Foundation (NSFC31271021 and NSFC31222023) and by the Distinguished Young Scholars of Sichuan University (2011SCU04B18).

1 Segatto, I., Berton, S., Sonega, M., Massarut, S., Andrea, S., Perin, T., Fabris, L., Armenia, J., Rampioni, G., Lovisa, S., Schiappacassi, M., Colombatti, A., Bristow, R., Vecchione, A., Baldassarre, G. \& Belletti, B. Inhibition of breast cancer local relapse by targeting p70S6 kinase activity. J. Mol. Cell. Biol. 5, 428-431 (2013).

2 Benson, J. \& Jatoi, I. The global breast cancer burden. Future Oncol. 8, 697-702 (2012).

3 Lin, Z., Gao, W., Hu, H., Ma, K., He, B., Dai, W., Wang, X., Wang, J., Zhang, X. \& Zhang, $Q$. Novel thermo-sensitive hydrogel system with paclitaxel nanocrystals: high drug-loading, sustained drug release and extended local retention guaranteeing better efficacy and lower toxicity. J. Control. Release 174, 161-170 (2014).

4 Desantis, C., Ma, J., Bryan, L. \& Jemal, A. Breast Cancer Statistics, 2013. Ca Cancer J. Clin. 64, 52-62 (2014).

5 Narod, S. Breast cancer in young women. Nat. Rev. Clin. Oncol. 9, 460-470 (2012).

6 Wöckel, A., Wolters, R., Wiegel, T., Novopashenny, I., Janni, W., Kreienberg, R., Wischnewsky, M. \& Schwentner, L. The impact of adjuvant radiotherapy on the survival of primary breast cancer patients: a retrospective multicenter cohort study of 8935 subjects. Ann. Oncol. 25, 628-632 (2014).

7 Kottke, T., Boisgerault, N., Diaz, R., Donnelly, O., Rommelfanger-Konkol, D., Pulido, J., Thompson, J., Mukhopadhyay, D., Kaspar, R., Coffey, M., Pandha, H., Melcher, A., Harrington, K., Selby, P. \& Vile, R. Detecting and targeting tumor relapse by its resistance to innate effectors at early recurrence. Nat. Med. 19, 1625-1630 (2013).

8 Pivot, X. Adjuvant chemotherapy for local relapse breast cancer. Lancet Oncol. 15, 125-126 (2014)

9 Yıldırım, E. Locoregional recurrence in breast carcinoma patients. EJSO $\mathbf{3 5}$, 258-263 (2009)

10 Waeber, M., Castiglione-Gertsch, M., Dietrich, D., Thürlimann, B., Goldhirsch, A. Brunner, K. \& Borner, M. Adjuvant therapy after excision and radiation of isolated postmastectomy locoregional breast cancer recurrence: definitive results of a phase III randomized trial (SAKK 23/82) comparing tamoxifen with observation. Ann. Oncol. 14, 1215-1221 (2003).

11 Orecchia, R. Breast cancer: Post-mastectomy radiotherapy reduces recurrence and mortality. Nat. Rev. Clin. Oncol. 11, 382-384 (2014).

12 Li, Y., Zou, L., Li, Q., Haibe-Kains, B., Tian, R., Li, Y., Desmedt, C., Sotiriou, C. Szallasi, Z., Iglehart, J., Richardson, A. \& Wang, Z. Amplification of ALPTM4B and YWHAZ contributes to chemotherapy resistance and recurrence of breast cancer. Nat. Med. 16, 214-218 (2010).

13 Huang, C., Steele, T., Widjaja, E., Boey, F., Venkatraman, S. \& Loo, J. The influence of additives in modulating drug delivery and degradation of PLGA thin films. NPG Asia Mater. 5, e54 (2013).

14 Lei, N., Gong, C., Qian, Z., Luo, F., Wang, C., Wang, H. \& Wei, Y. Therapeutic application of injectable thermosensitive hydrogel in preventing local breast cancer recurrence and improving incision wound healing in amouse model. Nanoscale 4, 5686-5693 (2012).

15 Aebi, S., Gelber, S., Anderson, S., Láng, I., Robidoux, A., Martín, M., Nortier, J., Paterson, A., Rimawi, M., Cañada, J., Thürlimann, B., Murray, E. \& Mamounas, E. Chemotherapy for isolated locoregional recurrence of breast cancer (CALOR): a randomised trial. Lancet Oncol. 15, 156-163 (2014).

$16 \mathrm{Li}$, J. Self-assembled supramolecular hydrogels based on polymer-cyclodextrin inclusion complexes for drug delivery. NPG Asia Mater. 26, 359-371 (2010).

17 Anseth, K., Bowman, C. \& Brannon-Peppas, L. Mechanical properties of hydrogels and their experimental determination. Biomaterials 17, 1647-1657 (1996).

18 Shin, S. \& Shea, L. Lentivirus immobilization to nanoparticles for enhanced and localized delivery from hydrogels. Mol. Ther. 18, 700-706 (2010).

19 Langer, R. \& Tirrell, D. Designing materials for biology and medicine. Nature $\mathbf{4 2 8}$, 487-492 (2004)

20 Wang, Y., Chen, L., Tan, L.i., Zhao, Q., Luo, Feng., Wei, Y. \& Qian, Z. PEG-PCL based micelle hydrogels as oral docetaxel delivery systemsfor breast cancer therapy. Biomaterials 35, 6972-6985 (2014).

21 Hennink, W. \& Nostrum, C. Novel crosslinking methods to design hydrogels. Adv. Drug Deliv. Rev. 64, 223-236 (2012).

22 Zhou, M., Singhana, B., Liu, Y., Huang, Q., Mitcham, T., Wallace, M., Stafford, R. Bouchard, R. \& Melancon, M. Photoacoustic- and magnetic resonance-guided photothermal therapy and tumor vasculature visualization using theranostic magnetic gold nanoshells. J. Biomed. Nanotechnol. 11, 1442-1450 (2015).

23 Zhou, F., Wu, S., Song, S., Chen, W., Resasco, D. \& Xing, D. Antitumor immunologically modified carbon nanotubes for photothermal therapy. Biomaterials 33, 3235-3242 (2012).

24 Peng, J., Qi, T., Liao, J., Chu, B., Yang, Q., Qu, Y, Li, W. Li, H., Luo, F. \& Qian, Z Mesoporous magnetic gold "nanoclusters" as theranostic carrier for chemo-photothermal co-therapy of breast cancer. Theranostics 47, 678-692 (2014).

25 Hirsch, L., Stafford, R., Bankson, J., Sershen, S., Rivera, B., Price, R., Hazle, J., Halas, N. \& West, J. Nanoshell-mediated near-infrared thermal therapy of tumors under magnetic resonance guidance. PNAS 100, 13549-13554 (2003). 
26 Peng, C., Shih, Y., Lee, P., Hsieh, T., Luo, T. \& Shieh, M. Multimodal image-guided photothermal therapy mediated by $188 \mathrm{Re}$-labeled micelles containing a cyanine-type photosensitizer. ACS Nano 5, 5594-5607 (2011).

27 Deng, H., Zhong, Y., Du, M., Liu, Q., Fan, Z., Dai, F. \& Zhang, X. Theranostic selfassembly structure of gold nanoparticles for NIR photothermal therapy and X-ray computed tomography imaging. Theranostics 4, 904-918 (2014).

28 Wang, S., Chen, K., Wu, T., Wang, H., Lin, W., Ohashi, M., Chiou, P. \& Tseng, H. Photothermal effects of supramolecularly assembled gold nanoparticles for the targeted treatment of cancer cells. Angew. Chem. Int. Ed. 49, 3777-3781 (2010).

29 Yavuz, M., Cheng, Y., Chen, J., Cobley, C., Zhang, Q., Rycenga, M., Xie, J., Kim, C., Song, K., Schwartz, A., Wang, L. \& Xia, Y. Gold nanocages covered by smart polymers for controlled release with near-infrared light. Nat. Mater. 8, 935-939 (2009).

30 Hribar, K., Lee, M., Lee, D. \& Burdick, J. Enhanced release of small molecules from near-infrared light responsive polymer-nanorod composites. ACS Nano $\mathbf{5}$, 2948-2956 (2011).

31 Matteini, B., Ratto, F., Rossi, F., Centi, S., Dei, L. \& Pini, R. Chitosan films doped with gold nanorods as laser-activatable hybrid bioadhesives. Adv. Mater. 22, 4313-4316 (2010).

32 Alkilany, A., Thompson, L., Boulos, S., Sisco, P. \& Murphy, C. Gold nanorods: their potential for photothermal therapeutics and drug delivery, tempered by the complexity of their biological interactions. Adv. Drug Deliv. Rev. 64, 190-199 (2012).

33 You, J., Zhang, G. \& Li, C. Exceptionally high payload of doxorubicin in hollow gold nanospheres for near-infrared light triggered drug release. ACS Nano 4 1033-1041 (2010).

34 Guo, W., Xia, H., Cao, L., Xia, F., Wang, S., Zhang, G., Song, Y., Wang, Y., Jiang, L. \& Zhu, D. Integrating ionic gate and rectifier within one solid state nanopore via modification with dual-responsive copolymer brushes. Adv. Funct. Mater. 20, 3561-3567 (2010)

35 Sau, T. \& Murphy, C. Seeded high yield synthesis of short Au nanorods in aqueous solution. Langmuir 20, 6414-6420 (2004).
36 Wang, K., Xu, X., Wang, Y., Yan, X., Guo, G., Huang, M., Luo, F., Zhao, X., Wei, Y. \& Qian, Z. Synthesis and characterization of poly(methoxyl ethylene glycolcaprolactone-co-methacrylic acid-co-poly(ethylene glycol) methyl ether methacrylate) $\mathrm{pH}$-sensitive hydrogel for delivery of dexamethasone. Int. J. Pharm. 389, 130-138 (2010).

37 Lee, J., Kim, H. \& Yoo, H. DNA nanogels composed of chitosan and Pluronic with thermo-sensitive and photo-crosslinking properties. Int. J. Pharm. 373 93-99 (2009).

38 Wang, K., Fu, S., Gu, Y., Xu, X., Dong, P., Guo, G., Zhao, X., Wei, Y. \& Qian, Z. Synthesis and characterization of biodegradable $\mathrm{pH}$-sensitive hydrogels based on poly (3-caprolactone), methacrylic acid, and poly(ethylene glycol). Polym. Degrad. Stab. 94, 730-737 (2009).

39 Gong, C., Wang, C., Wang, Y., Wu, Q., Zhang, D., Luo, F. \& Qian, Z. Efficient inhibition of colorectal peritoneal carcinomatosis by drug loaded micelles in thermosensitive hydrogel composites. Nanoscale 4, 3095-3104 (2012).

40 Yang, B., Li, X., Shi, S., Kong, X., Guo, G., Huang, M., Luo, F., Wei, Y., Zhao, X. \& Qian, Z. Preparation and characterization of a novel chitosan scaffold. Carbohyd. Polym. 80, 860-865 (2010).

(a) (i) This work is licensed under a Creative Commons Attribution 4.0 International License. The images or other third party material in this article are included in the article's Creative Commons license, unless indicated otherwise in the credit line; if the material is not included under the Creative Commons license, users will need to obtain permission from the license holder to reproduce the material. To view a copy of this license, visit http:// creativecommons.org/licenses/by/4.0/

Supplementary Information accompanies the paper on the NPG Asia Materials website (http://www.nature.com/am) 